FORMATION Formation emploi

Revue française de sciences sociales

137 | Janvier-Mars 2017

La formation continue en contexte : l'entreprise au cœur des enjeux

\title{
Introduction : La formation continue en contexte : l'entreprise et ses salariés au cœur des enjeux
}

Arnaud dupray, Danielle Guillemot et Ekaterina Melnik-Olive

\section{OpenEdition}

Journals

Édition électronique

URL : http://journals.openedition.org/formationemploi/5031

DOI : 10.4000/formationemploi.5031

ISSN : 2107-0946

Éditeur

La Documentation française

Édition imprimée

Date de publication : 30 avril 2017

Pagination : 7-14

ISSN : 0759-6340

Référence électronique

Arnaud dupray, Danielle Guillemot et Ekaterina Melnik-Olive, « Introduction : La formation continue en contexte : l'entreprise et ses salariés au cœur des enjeux ». Formation emploi [En ligne], 137 | JanvierMars 2017, mis en ligne le 30 avril 2017, consulté le 30 octobre 2020. URL : http://

journals.openedition.org/formationemploi/5031 ; DOI : https://doi.org/10.4000/formationemploi.5031

(C) Tous droits réservés 


\title{
Introduction
}

\section{La formation continue en contexte : l'entreprise et ses salariés au cœur des enjeux}

\author{
Arnaud Dupray \\ Economiste, chargé d'études au Céreq, membre associé à Aix-Marseille Univ., CNRS, LEST, \\ Aix-en-Provence, France \\ Danielle Guillemot \\ Responsable du Département Formation et Certification au Céreq \\ Ekaterina MeLnik-Olive \\ Chargée d'études au Céreq, membre associée à Aix-Marseille Univ., CNRS, LEST, Aix-en- \\ Provence, France
}

De longue date, l'éducation et la formation, notamment la formation professionnelle continue (FPC), sont conçues, dans les économies occidentales et par les organismes internationaux, comme des piliers du développement économique. Elles concourent à la montée en qualification de la main-d'œuvre et à sa capacité d'adaptation face aux transformations rapides des technologies, des modes d'échange et de production (Cedefop, 2010). Experts, pouvoirs publics ou partenaires sociaux assignent également à la FPC un rôle clé dans l'intégration économique et sociale des citoyens et dans la réduction des inégalités sur le marché du travail (Gaussel, 2011). En particulier, l'idée selon laquelle la formation serait le meilleur rempart contre le chômage est largement partagée (Méhaut, 1996).

En dépit de cette belle unanimité (Merle, 2006), on constate d'année en année que, en France comme dans la plupart des pays occidentaux, le système de FPC souffre de défauts récurrents. Ainsi, les salariés les moins diplômés, moins qualifiés, plus précaires accèdent le moins à la formation en entreprise (Perez, 2009). De même, les moins qualifiés expriment le moins le besoin de se former (Fournier, 2004 ; Fournier 2006). Pourtant, ces catégories constituent la cible prioritaire des politiques publiques de sécurisation des parcours professionnels.

En effet, concilier les divers objectifs assignés à la FPC par les différents acteurs ne va pas de soi. Les entreprises forment le plus souvent pour répondre aux impératifs de la production et aux changements organisationnels et techniques (Dubois et al., 2016) ; cela ne coïncide pas toujours avec les objectifs de salariés qui cherchent à améliorer leur situation professionnelle, leur qualification ou à changer de métier, tandis que les pouvoirs publics souhaitent notamment orienter les moins qualifiés et les demandeurs d'emploi vers les " métiers en tension » ou "d'avenir ". 
Selon l'approche économique standard, les entreprises financent une formation si elles en attendent un retour, au travers de l'augmentation de la productivité du salarié. Mais elles limiteront l'investissement dans la formation des salariés s'il y a un risque, qu'une fois formés, ceux-ci partent travailler dans d'autres entreprises. Elles peuvent aussi préférer se comporter en " passager clandestin " en recrutant des salariés déjà formés plutôt que de supporter les coûts d'une formation transférable d'une entreprise à l'autre.

Or, le besoin en personnel qualifié conduit les entreprises à s'organiser, notamment par branche d'activité, pour mutualiser des dispositifs de formation susceptibles de bénéficier à l'ensemble des entreprises de la branche ; dans ce cas, c'est l'organisation professionnelle ou les partenaires sociaux, les syndicats de salariés cherchant à promouvoir la qualification de leurs mandants, qui participent à la régulation pour améliorer l'investissement global en formation. Ainsi, des formes de régulation s'imposent, même dans les pays les plus enclins à laisser le " marché » décider des investissements en FPC.

Si en France, comme dans beaucoup d'autres pays, les pouvoirs publics et les organisations professionnelles ou paritaires jouent bien un rôle régulateur, la faiblesse des investissements en formation continue, dans certains segments de l'économie, et la persistance des inégalités d'accès montrent bien les difficultés de cette régulation.

La problématique de la régulation de la formation peut se décliner aussi au niveau de l'entreprise avec son contexte, ses pratiques managériales ou encore les modalités du dialogue social mises en place. Les décisions des entreprises en matière de formation peuvent s'appuyer sur une rationalité plus complexe que le seul retour immédiat sur investissement, prenant en compte diverses dimensions, comme par exemple les perspectives de développement à long terme ou encore la motivation des salariés. La formation peut être mobilisée pour être le support de la mise en œuvre du partage d'une culture professionnelle ou de valeurs d'entreprise. À l'opposé, elle peut préparer des ruptures professionnelles et contribuer à amortir le coût d'un plan social. Ainsi, les objectifs assignés à la formation continue en entreprise peuvent être multiples : accroître l'efficacité, favoriser l'innovation, répondre aux obligations légales, accompagner le changement, motiver et fidéliser ses salariés, faciliter les mobilités, ou encore améliorer l'image de l'entreprise.

Dans un esprit pluridisciplinaire, ce dossier réunit des travaux mobilisant des approches issues de la science politique, de l'économie et de la socio-économie. Les textes apportent un regard renouvelé sur la question de la régulation sociale de la formation continue. Au travers d'investigations empiriques qualitatives et quantitatives originales, ces questions sont abordées sous deux angles différents. Le premier met l'accent sur le contexte sociétal et la variété des enjeux des acteurs impliqués. Les formes de régulation de la formation passent d'abord par son financement, la nature des différents acteurs pouvant y participer, mais aussi les modalités de coordination entre organismes et acteurs qui gèrent les fonds. Le second angle s'intéresse à la façon dont ces régulations se déclinent dans les pratiques de formation des entreprises, avec un accent mis sur l'organisation et 
son contexte. Ici, le questionnement porte sur les stratégies et les comportements des entreprises en matière de FPC, analysés dans le cadre élargi des pratiques de gestion des ressources humaines et des compétences.

\section{Les acteurs de la régulation de la formation continue}

Les questions de régulation d'ordre systémique sont abordées dans les deux premières contributions : la première, sur le système de financement de la formation continue en Suisse (I. Voirol-Rubido), la suivante, à travers une comparaison de la gouvernance des systèmes paritaires de collecte et de gestion de fonds pour la FPC, en France et en Italie (J. Grabener). S'appuyant sur des entretiens qualitatifs auprès d'acteurs de la FPC dans une approche de science politique, ces deux premières contributions mettent en évidence la diversité des enjeux de régulation du financement de la FPC des salariés.

Cette question, étudiée par Isabel Voirol-Rubido dans le cas de la Suisse, est une affaire essentiellement privée. Le financement de la formation est pris en charge par l'entreprise ou l'individu. La moitié environ de l'effort de formation est assumée par les formés. Si les taux d'accès des actifs occupés sont dans une bonne moyenne (proche de ce que l'on observe en France), les inégalités sont, comme ailleurs, importantes, au détriment des publics les plus précarisés.

Ce constat préoccupe les pouvoirs publics, qui jugent la situation préjudiciable à la compétitivité des entreprises et à la croissance du pays. Ils cherchent ainsi à soutenir l'investissement en formation en direction des individus les moins bien lotis.

L'originalité de la recherche relatée dans cet article réside dans la présentation d'un modèle de financement - inspiré notamment des recommandations de l'OCDE (Organisation de coopération et de développement économiques) - aux principaux acteurs de la formation continue, dans le but d'en tester la faisabilité politique. Dans le cas de la Suisse, le patronat est opposé à l'intervention des pouvoirs publics dans la formation. Il refuse en effet « l'ingérence de l'État » dans les décisions de l'entreprise, qui doit être seule habilitée à gérer le recours à la formation en fonction de ses besoins. Pour cet acteur central, le financement public ouvrirait la porte à un processus de contrôle qui entraverait la flexibilité et la réactivité de l'offre de formation et conduirait probablement à sa standardisation. Les milieux économiques sont cependant favorables à ce que l'État développe et finance une offre de formation pertinente à destination des publics les moins qualifiés, marché délaissé par le privé car considéré comme économiquement peu rentable. Les syndicats partagent une certaine méfiance vis-à-vis de l'intervention de l'État. Mais surtout, ils jugent impossible d'instaurer ce type de modèle sur le plan politique en raison de la très forte influence du patronat, qui s'y opposerait. En revanche, pour les syndicats, le renforcement d'un financement bipartite (employeur/ 
salarié) de la formation continue au travers des Conventions Collectives de Travail, qui présentent l'avantage d'être indépendantes des pouvoirs publics, pourrait constituer un vecteur prometteur pour promouvoir la formation continue des publics prioritaires.

Josua Gräbener s'intéresse au financement de la FPC par le biais des organismes collecteurs des fonds de la formation qui existent en France et en Italie. L'article interroge l'inégale efficacité d'organismes gérés par les partenaires sociaux dans ces deux pays, proches en apparence. Les "Fondi Paritetici Interprofessionnali " (FPI), largement inspirés des "Organismes Paritaires Collecteurs Agréés » (OPCA) français, se distinguent de ces derniers en ce qu'ils évoluent dans un environnement fortement concurrentiel. En France, les accords interprofessionnels transcrits dans la loi, ainsi que les accords de branches contraignent les entreprises à verser leurs cotisations aux OPCA. En revanche, en Italie, les employeurs décident s'ils souhaitent verser leurs cotisations à un FPI et font un choix d'affectation. Et contrairement aux OPCA, gérés par les partenaires sociaux des branches professionnelles sous-traitant une mission de service public, les FPI sont en concurrence pour obtenir les cotisations des entreprises. Si bien que ces dernières doivent faire face à une forte instabilité de leurs ressources et cherchent à satisfaire rapidement le plus grand nombre possible d'entreprises en leur attribuant facilement des (petits) financements pour la FPC des salariés. En particulier, le système est peu favorable au financement de formations certifiantes visant à sécuriser les parcours professionnels des salariés. Pour l'auteur, les «pressions contributives » dues à la gouvernance par le marché constituent un important élément explicatif de ces différences entre les OPCA et les FPI.

Le décentrage opéré par l'analyse de systèmes étrangers permet ainsi de mettre en évidence le rôle central du fonctionnement des institutions, fortement dépendant du jeu des acteurs et de leurs préoccupations, y compris lorsque les systèmes de financement de la formation sont à première vue très proches. Dans le domaine de la formation continue, les positions de l'acteur patronal sont centrales, foncièrement libérales en Suisse, fortement organisées en branches professionnelles en France, ou plus atomisées en Italie. Grâce à un regard comparatif, replacé dans des contextes institutionnels et culturels différents, le système français est ainsi questionné.

\section{Les régulations dans les pratiques de formation des entreprises}

En France, depuis la loi de 1971, l'entreprise, lieu de réalisation du droit à la formation des salariés, demeure l'acteur majeur de la FPC. La participation des salariés à la formation est ainsi marquée par les caractéristiques des entreprises qui la mettent en oeuvre.

Les questions de régulations de la FPC au niveau des entreprises sont explorées dans les quatre autres articles du dossier. Ceux-ci interrogent la réalité française à travers 
l'examen des pratiques au sein des entreprises. Comment celles-ci mobilisent-elles la formation en fonction de la conjoncture et de la dynamique de l'activité économique ? Dans quelle mesure leurs modes d'organisation et de management internes élargissentils les opportunités de développement et d'usage de la formation continue par les salariés ? Quels sont les impacts sur les inégalités d'accès selon les modes de gestion de la FPC et son intégration dans les outils de la GRH ? Comment rendre compte d'usages de la formation en entreprise lorsque ceux-ci semblent aller à l'encontre des hypothèses théoriques de retour sur investissement ?

Ces quatre articles utilisent des méthodes quantitatives et s'appuient sur l'exploitation du dispositif d'enquêtes couplées employeurs-salariés DIFES2, mis en place et conduit par le Céreq en 2012. Plus de 1800 entreprises d'au moins dix salariés et plus de 6000 salariés employés dans ces entreprises - représentatifs de plus de 13 millions de salariés présents dans les entreprises du secteur marchand en 2010 - ont été interrogés. À l'origine de nombreux travaux du Céreq autour de la FPC (Lambert et al. 2015), ce dispositif a également fait l'objet d'un groupe de travail qui a réuni des chargés d'études du Céreq et plusieurs équipes universitaires, dont sont issues, notamment, ces contributions. Le double regard sur les dimensions individuelles de la formation et le contexte de l'entreprise dans laquelle le salarié est formé, apporté par ce dispositif, a permis d'aborder une variété de questions autour du lien entre formation continue et parcours professionnels des salariés, avec un accent mis sur le rôle des contextes organisationnel et économique.

M. Guergoat et C. Perez analysent le recours à la formation par les entreprises françaises en lien avec les dynamiques de leurs activités économiques. Les auteures interrogent ainsi la sensibilité à la conjoncture économique du recours à la formation continue par les entreprises, dès lors qu'elle s'apparente à un détour de production. D'un point de vue théorique, la relation entre la variation de l'activité de l'entreprise et son effort de formation n'est pas déterminée de manière univoque. Si l'entreprise considère la formation des salariés comme un investissement, la baisse d'activité peut être une occasion, pour celle-ci, de profiter de faibles coûts d'opportunité (pertes en production ou nécessité de remplacer le salarié) liés à l'absence du salarié parti en formation. Mais à l'inverse, la baisse d'activité peut avoir un impact négatif sur la formation, si celle-ci est considérée davantage comme un coût à réduire. L'article permet de préciser la nature de ces relations entre les fluctuations de l'activité et la formation en entreprise, en identifiant une variété de contextes économiques et de modes d'ajustement. Sans exclure l'existence de ces deux effets opposés, qui peuvent coexister, les observations empiriques mettent en évidence le caractère favorable d'une certaine stabilité ou visibilité de l'horizon économique pour l'investissement en formation.

La prise en compte du contexte propre à l'environnement économique de l'entreprise a permis ici d'aborder la question de la formation sous les aspects dynamique et quantitatif. Les trois contributions suivantes offrent l'opportunité de saisir les modalités 
internes de mise en œuvre de la formation, lesquelles vont affecter les manières dont celle-ci se décline qualitativement (apport en congruence avec les aspirations des salariés, levier de mobilités,...).

Le texte de J. Vero et J.-C. Sigot questionne le rôle de l'organisation dans la mise en place des conditions permettant aux salariés de devenir acteurs de leurs propres parcours professionnels. Les pratiques des entreprises sont analysées à l'aune de la théorie des capacités de A. Sen. Les auteurs distinguent les types de configurations organisationnelles en fonction de quatre dimensions structurantes de l'espace des possibles : la diversité des objectifs assignés à la formation par l'entreprise, les opportunités offertes en termes de formation, les formes de participation au dialogue en entreprise, la pluralité des réalisations en termes de formation pour le salarié (au-delà de la simple adaptation au poste). Le rôle de la formation en entreprise dans la sécurisation des parcours des salariés renvoie à l'idée que la formation doit s'inscrire dans un objectif qui satisfasse à la fois le salarié et l'entreprise. Suivant sa rationalité économique, l'entreprise cherche en effet à ce que la formation rejoigne ses objectifs. Les organisations " capacitantes " seraient alors ces organisations vertueuses où il est possible pour le salarié d'engager une formation qui réponde aussi à son propre objectif professionnel.

Les pratiques vertueuses en matière de gestion des compétences et de la formation en entreprise sont également mises en discussion par le texte d'E. Melnik-Olive et H. Couprie. L'article analyse les différences d'accès à la formation et à la promotion en entreprise selon le genre, en fonction des outils managériaux et des pratiques de formation dans les entreprises. À la suite de travaux déjà anciens (Béret et Dupray, 1998) les auteures ne cherchent pas à identifier une relation de causalité entre formation et promotion, les deux mouvements étant le plus souvent concomitants. En revanche, une attention renouvelée est accordée, dans cet article, au contexte organisationnel dans lequel les salariée.e.s se forment (ou pas) et peuvent (ou non) évoluer. Les auteures identifient trois clés de lecture permettant de décrire le contexte organisationnel favorable à l'égalité des chances de promotion entre les hommes et les femmes : la transparence de l'information, la réflexion structurée autour des enjeux de la formation et du développement des compétences, et le dialogue avec les salariés et leurs représentants. Il ressort des analyses une différenciation dans la façon dont les salarié.e.s accèdent à la formation et à la promotion. Des mécanismes de sélection différents peuvent en effet être mis au jour selon que la formation représente une nécessité (en termes d'adaptation au poste) ou un outil d'incitation (fidélisation, motivation) des salariés présélectionnés. C’est dans cette dernière logique que promotion et formation sont le plus associées. En outre, l'article souligne que les inégalités d'accès à la promotion en entreprise, observées entre les femmes et les hommes, sont considérablement réduites par des pratiques d'entreprises répondant simultanément aux critères de transparence, de réflexion stratégique et de dialogue autour de la formation.

Dans la continuité de l'article précédent, mais dans une optique différente, le texte de B. Cart, V. Henguelle et M.-H. Toutin relève des usages différenciés de la formation par 
les entreprises, réinterrogés à l'aune de la théorie beckerienne du capital humain. Cette théorie économique met en avant le risque de sous-investissement des entreprises dans la formation de leurs salariés, si ceux-ci peuvent la valoriser ailleurs. Ce risque est d'autant plus important que les compétences acquises sont transférables. Dans ce cadre théorique, on s'attend à une certaine stabilité de la relation d'emploi des salariés ayant bénéficié d'une formation, en particulier lorsqu'elle est spécifique et prise en charge par l'entreprise. L'analyse confirme que le suivi d'une formation, quand elle est spécifique, rend moins probable le départ de l'entreprise. Cependant, on observe des départs (démissions et ruptures de contrat) de salariés ayant bénéficié d'une formation, y compris spécifique. Les auteurs tentent alors de comprendre les motifs de ces départs allant, à première vue, à l'encontre des prédictions théoriques. Il ressort de leur analyse que l'effet " protecteur " de la formation spécifique semble moins concerner les salariés ayant exprimé des besoins de formation insatisfaits. Ce sont aussi ceux qui n'ont pas obtenu d'augmentation salariale, qui ont pris en charge une partie de leur formation ou encore dont les conditions de travail sont pénibles. En revanche, les salariés ayant bénéficié d'une formation en lien avec leur projet professionnel ont moins de chances de quitter leur entreprise.

Il ressort de cette analyse une diversité des usages de la formation en entreprise, qui peuvent être plus ou moins en rapport avec les attentes des salariés. Ainsi, la participation des salariés à la formation continue peut s'effectuer selon des objectifs qui ne convergent pas nécessairement, entre le souhait de l'entreprise, visant à la rentabiliser, et les attentes du salarié en matière de progression professionnelle. De plus, ces attentes peuvent différer selon les salariés et porter tantôt sur une évolution au sein de l'entreprise, un gain en qualification ou encore sur le souhait d'améliorer ses conditions de travail, y compris en changeant d'emploi ou de métier.

Les résultats présentés dans ce dossier ont permis d'aborder la formation continue des salariés en la mettant en relation avec le contexte sociétal, conjoncturel ou organisationnel. Ils invitent à revenir sur les enjeux de la formation continue en termes de sa régulation sociale et en relation avec les objectifs qui lui sont assignés par les différents acteurs. Mais ces contributions laissent de côté certaines zones d'ombre : son articulation avec des formations initiales de plus en plus professionnalisées, mais aussi avec d'autres situations d'apprentissage (échanges informels, activités au travail), ses effets en matière de sécurisation des parcours professionnels, l'usage de la formation professionnelle continue dans les très petites entreprises (TPE)...

Éclairer ces zones d'ombre fait partie de l'ambition du nouveau Dispositif d'Enquêtes sur les Formations et Itinéraires des Salariés (DEFIS), actuellement conduit par le Céreq. Ce dispositif a été lancé en 2015, à la demande des partenaires sociaux. S'appuyant sur la mise en lien de données Entreprises-Salariés, DEFIS intègre un suivi longitudinal des parcours individuels, avec un accent mis notamment sur les descriptions des formations suivies et changements professionnels vécus. L'acception de la formation élargie au-delà de la formation organisée, le repérage des contextes favorables aux apprentissages au travail, 
le champ de l'enquête étendu aux entreprises d'au moins trois salariés constituent autant d'éléments nouveaux pour améliorer la connaissance de la formation continue en entreprise, en France. Nul doute que ce nouveau dispositif permettra de prolonger et d'approfondir les analyses développées dans ce dossier.

\section{Bibliographie}

Béret P., Dupray A. (1998), « La formation professionnelle continue : de l'accumulation de compétences à la validation de la performance ", Formation Emploi, Vol. 63, n 1, pp. 61-80.

Cedefop (2010), A bridge to the future European policy for vocational education and training 2002-10, Cedefop Reference Series.

Dubois J.-M., Marion-Vernoux I., Noack E. (2016), « Le dispositif d'enquête Defis, un nouveau regard sur la formation en entreprise ", Céreq-Bref, n 344 , mars, 4 p.

Fournier Ch. (2004), " Aux origines de l'inégale appétence des salariés pour la formation ", Céreq-Bref, n² 209, juin.

Fournier Ch. (2006) «Les besoins de formation non-satisfaits des salariés au prisme des catégories sociales " Formation Emploi, n 95 , juillet-septembre.

Gaussel M. (2011), "Se former tout au long de sa vie d'adulte », IFE, Veilles et Analyses $\mathrm{n}^{\circ} 61$, avril.

Lambert M., Marion-Vernoux I., Sigot J.-C., Vero J. (2015), « Bilan du dispositif d'information sur la formation employeurs-salariés ", Net.Doc Céreq, n 146, décembre, 187 p.

Méhaut P. (1996), «Se former tout au long de la vie ?», Céreq-Bref, n 120, avril.

Merle V. (2006), « La formation tout au long de la vie : un projet pour les sociétés démocratiques ", in Morvan Y. (dir.), La formation tout au long de la vie. Nouvelles questions, nouvelles perspectives, Presses universitaires de Rennes, pp. 23-37.

Perez C. (2009), « Pourquoi les travailleurs précaires ne participent-ils pas à la formation professionnelle continue?» Formation Emploi, n 105, janvier-mars. 